\title{
Gauged and Ungauged Unparticles Signal at the LHC and ILC
}

\section{N.Mebarki ${ }^{1}$}

Laboratoire de Physique Mathematique et Subatomique, Mentouri University

Route Ain El Bey, Constantine 25000, Algeria

E-mail: nnmebarki@yahoo.fr

\section{I.Aliane}

Laboratoire de Physique Mathematique et Subatomique, Mentouri University

Route Ain El Bey, Constantine 25000, Algeria

E-mail: thouguer@yahoo.fr

\begin{abstract}
A novel scalar and tensor unparticles contribution to the polarized diphoton production at the $\mathrm{LCH}$ and ILC are studied and the effects of the conformal as well dimensions scales are discussed. The expression of the various subprocess helicity amplitudes is also presented. Moreover, the effect of spin 0 and spin $1 / 2$ gauged unparticles on the decay rates $h \rightarrow \gamma \gamma$ and $h \rightarrow Z \gamma$ are also shown explicitly.
\end{abstract}

Frontiers of Fundamental Physics 14

FFP14

15-18 July 2014

Aix Marseille University (AMU) Saint-Charles Campus, Marseille 


\section{Introduction}

Conformal symmetry plays an important role in both critical phenomena and superstring theory. However, in particle physics and in four dimensional space-time it is broken explicitly by the masses of the particles. At high energies, they could exist stuff with non trivial scale invariance in the infrared regime such as the Banks-Zaks fields which are recently suggested by Howard Georgi as a new component beyond the standard Model (SM) above the TeV scale [1][2]. Georgi has used the method of the low energy effective field theory to study the unparticle stuff production and the peculiar virtual effects in high energy processes. The goal of this paper is to study the contribution of scalar and tensor unparticles to the helicity amplitudes of the diphoton production at the LCH and ILC and the effects of the conformal dimensions scales. Moreover, the effect of spin 0 and spin $1 / 2$ gauged unparticles on the decay rates $h \rightarrow \gamma \gamma$ and $h \rightarrow Z \gamma$ are also shown explicitly. In section 2, we present the theoretical calculations and some phenomenology. Finally in section 3 , we make a discussion and draw our conclusion.

\section{The model}

In the standard model, at the leading order in perturbative QCD, the diphoton production is dominated by the quark-antiquark annihilation subprocess $q \bar{q} \rightarrow \gamma \gamma$ and the gluon-gluon fusion subprocess $g g \rightarrow \gamma \gamma$ via a box virtual quarks diagram. Because of the dominance of the gluon distribution function (luminosity) inside the proton at the LHC energies, the subprocess $g \bar{g} \rightarrow \gamma \gamma$ becomes important.This gives a possibility to study the unparticles interactions allowing the couplings of the gluons and photons at the tree level. At the ILC, the subprocess $\gamma \gamma \rightarrow \gamma \gamma$ is also very important. Regarding the theoretical helicity transition amplitudes, for the subprocess $g \bar{g} \rightarrow \gamma \gamma$, straightforward but tedious calculations lead to the following expressions:

$$
\begin{gathered}
\left|M_{u_{s}}^{h_{1}, h_{2}, h_{3}, h_{4}}\right|^{2}=C_{s}^{2}\left(N_{c}^{2}-2\right) C_{F} \hat{s}^{2 d_{u_{s}}}\left[\left(h_{1} h_{2}+1\right)^{2} \lambda_{s, g}^{2}+\left(h_{1}+h_{2}\right)^{2} \lambda_{s, g}^{2}\right]\left[\left(h_{3} h_{4}+1\right)^{2} \lambda_{s, \gamma}^{2}+\right. \\
\left.\left(h_{3}+h_{4}\right)^{2} \lambda_{s, \gamma}^{\prime 2}\right] /\left[N_{c} \Lambda_{u}^{4 d_{u_{s}}}\right] \\
\left|M_{u_{t}}^{h_{1}, h_{2}, h_{3}, h_{4}}\right|^{2}=C_{t}^{2} \hat{s}^{2 d_{u_{s}} \lambda_{s, \gamma}^{2}}\left[\left(N_{c}^{2}-2\right) C_{F} \lambda_{t, g}^{2}+N_{c}^{2} C_{F} \lambda_{t, g}^{2}\right]\left[( h _ { 1 } h _ { 2 } - 1 ) ( h _ { 3 } h _ { 4 } - 1 ) \left(\hat{t}^{2}+\right.\right. \\
\left.\left.\hat{u}^{2}\right)-\left(h_{1}-h_{2}\right)\left(h_{3}-h_{4}\right)\left(\hat{t}^{2}-\hat{u}^{2}\right)\right]^{2} /\left[64 N_{c} \hat{s}^{4} \Lambda_{u}^{4 d_{u_{s}}}\right]
\end{gathered}
$$

where $h_{i},(i=\overline{1,4})$ stands for the helecity of the $i$ th particle, $\lambda_{s, g}$ and $\lambda_{t, g}$ are the scalar and tensor couplings with the gluon $\left(\boldsymbol{C}_{\boldsymbol{F}}\right.$ and $\boldsymbol{N}_{\boldsymbol{c}}$ are $S U\left(N_{c}\right)$ color factors). For the subprocess $\gamma \gamma \rightarrow \gamma \gamma$, we obtain:

$$
\begin{gathered}
\left|M_{u_{s}}^{(s) \pm \pm}\right|^{2}=2 \Omega(\hat{s}, \hat{s}) / \cos \left(\pi d_{u_{s}}\right),\left|M_{u_{s}}^{(t) \pm \pm}\right|^{2}=\left|M_{u_{s}}^{(t) \pm \mp}\right|^{2}=\Omega(\hat{t}, \hat{t}) / \cos \left(\pi d_{u_{s}}\right) \\
\left|M_{u_{s}}^{(u) \pm \pm}\right|^{2}=\left|M_{u_{s}}^{(u) \pm \mp}\right|^{2}=\Omega(\hat{u}, \hat{u}) / \cos \left(\pi d_{u_{s}}\right) \\
\left|M_{u_{s}}^{(s) \pm \mp}\right|^{2}=\operatorname{Re}\left(M_{u_{s}}^{s *} M_{u_{s}}^{u}\right)^{ \pm \mp}=\Re e\left(M_{u_{s}}^{t *} M_{u_{s}}^{u}\right)^{ \pm \mp}=\Re e\left(M_{u_{s}}^{s *} M_{u_{s}}^{t}\right)^{ \pm \mp}=0 \\
\Re e\left(M_{u_{s}}^{t *} M_{u_{s}}^{u}\right)^{ \pm \pm}=\Omega(\hat{u}, \hat{t}), \Re e\left(M_{u_{s}}^{s *} M_{u_{s}}^{u}\right)^{ \pm \pm}=\Omega(\hat{s}, \hat{u}), \quad \Re e\left(M_{u_{s}}^{s *} M_{u_{s}}^{t}\right)^{ \pm \pm}=\Omega(\hat{s}, \hat{t})
\end{gathered}
$$




$$
\begin{gathered}
\left|M_{u_{t}}^{(s) \pm \mp}\right|^{2}=\widetilde{\Omega}(\hat{s}, \hat{s}, \hat{u})+\widetilde{\Omega}(\hat{s}, \hat{s}, \hat{t}),\left|M_{u_{t}}^{(u) \pm \pm}\right|^{2}=\widetilde{\Omega}(\hat{u}, \hat{u}, \hat{t})+\widetilde{\Omega}(\hat{u}, \hat{u}, \hat{s}) \\
\left|M_{u_{t}}^{(t) \pm \pm}\right|^{2}=\widetilde{\Omega}(\hat{t}, \hat{t}, \hat{u})+\widetilde{\Omega}(\hat{t}, \hat{t}, \hat{s}),\left|M_{u_{t}}^{(t) \pm \mp}\right|^{2}=\left|M_{u_{t}}^{(u) \pm \mp}\right|^{2}=\left|M_{u_{t}}^{(s) \pm \pm}\right|^{2}=0 \\
\Re e\left(M_{u_{t}}^{s *} M_{u_{t}}^{t}\right)^{ \pm \pm}=\Re e\left(M_{u_{t}}^{s *} M_{u_{t}}^{u}\right)^{ \pm \pm}=\Re e\left(M_{u_{t}}^{t *} M_{u_{t}}^{u}\right)^{ \pm \mp}=0
\end{gathered}
$$

$\Re e\left(M_{u_{t}}^{s *} M_{u_{t}}^{t}\right)^{ \pm \mp}=\widetilde{\Omega}(\hat{s}, \widehat{t}, \hat{u}), \quad \Re e\left(M_{u_{t}}^{s *} M_{u_{t}}^{u}\right)^{ \pm \pm}=\widetilde{\Omega}(\hat{s}, \hat{u}, \hat{t}), \quad \Re e\left(M_{u_{t}}^{t *} M_{u_{t}}^{u}\right)^{ \pm \pm}=\widetilde{\Omega}(\hat{u}, \hat{t}, \hat{s})(10)$ where

$$
\begin{array}{r}
\Omega(\hat{s}, \hat{t})=4 C_{s}^{2}\left[\lambda_{s, \gamma}^{2}+4 \lambda_{s, \gamma}^{\prime 2}\right]^{2} \hat{s}^{d_{u_{s}}} \hat{t}^{d_{u_{s}} \cos \left(\pi d_{u_{s}}\right) / \Lambda_{u}^{4 d_{u_{s}}}} \\
\widetilde{\Omega}(\hat{s}, \hat{t}, \hat{u})=C_{t}^{2} \lambda_{t, \gamma}^{4} \hat{s}^{d_{u_{t}}-2} \hat{t}^{d_{u_{t}}-2} \hat{u}^{4} \cos \left(\pi d_{u_{t}}\right) /\left(2 \Lambda_{u}^{4 d_{u_{t}}}\right) \\
C_{s}=A\left(d_{u_{s}}\right) /\left[2 \sin \left(\pi d_{u_{s}}\right)\right], \quad C_{t}=A\left(d_{u_{t}}\right) /\left[2 \sin \left(\pi d_{u_{t}}\right)\right]
\end{array}
$$

and

$$
A(n)=\left[16 \pi^{\frac{5}{2}} \Gamma(n+1 / 2)\right] /\left[\left(2 \pi^{2 n} \Gamma(n-1) \Gamma(2 n)\right]\right.
$$

$\Lambda_{u}$ plays the role of an ultra violet cut of (UV) of the effective theory, $\lambda_{i}$ and $\lambda_{i}^{\prime}$ are dimensionless effective couplings constants.

Regarding the Higgs diphoton and $Z \gamma$ decays $h \rightarrow \gamma \gamma$ and $h \rightarrow Z \gamma$ respectively with gauged scalar and fermion unparticles loops, Direct calculations gives the following expressions of the ratios $R_{\gamma \gamma}$ and $R_{Z \gamma}$ :

and

$$
R_{\gamma \gamma} \approx \frac{\Gamma(h \rightarrow \gamma \gamma)_{\mathrm{MS}+\mathrm{NP}}}{\Gamma(h \rightarrow \gamma \gamma)_{\mathrm{MS}}}=\left|1-\frac{Q_{u_{S}}^{2} v^{2} \lambda_{h u_{S}} A_{u_{S}}^{\gamma \gamma}}{\left[2\left(\mu^{2}\right)^{2-d_{u_{S}} \Lambda_{u}^{2 d_{u_{S}}-2}} A_{S M}^{\gamma \gamma}\right]}-\frac{Q_{u_{f}}^{2} v^{2} \lambda_{h u_{f}} A_{u_{f}}^{\gamma \gamma}}{\left[2\left(\mu^{2}\right)^{2-d_{u_{f}}} \Lambda_{u}^{2 d_{u_{f}}{ }^{-2}} A_{S M}^{\gamma \gamma}\right]}\right|^{2}
$$

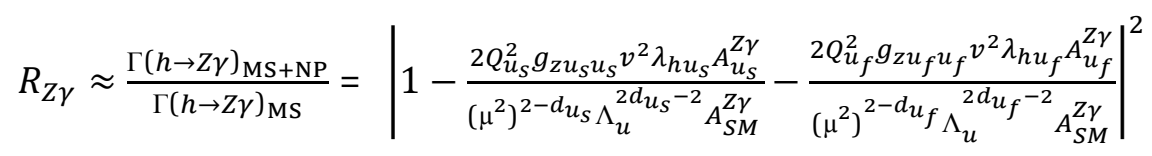

where $\mu$ (resp. $d_{u_{i}}$ ) is the conformal symmetry breaking scale (resp. scale dimension) and

$$
\begin{gathered}
A_{S M}^{\gamma \gamma}=A_{W}\left(\tau_{W}\right)+N_{c} Q_{\text {top }}^{2} A_{\text {top }}\left(\tau_{\text {top }}\right) \\
A_{S M}^{Z \gamma}=\cot \theta_{w} A_{W}^{Z \gamma}\left(\tau_{W}, \lambda_{W}\right)+2 Q_{\text {top }} N_{c}\left(T_{3}^{\text {top }}-2 Q_{\text {top }} s_{w}^{2}\right) A_{\text {top }}^{Z \gamma}\left(\tau_{\text {top }}, \lambda_{\text {top }}\right) / c_{w} s_{w} \\
A_{\text {top }}\left(\tau_{\text {top }}\right)=3\left[\tau_{\text {top }}+\left(\tau_{\text {top }}-1\right) L\left(\tau_{\text {top }}\right)\right] /\left(2 \tau_{\text {top }}^{2}\right) \\
A_{W}\left(\tau_{W}\right)=-\left[3 \tau_{W}+2 \tau_{W}^{2}+3\left(2 \tau_{W}-1\right) L\left(\tau_{W}\right)\right] /\left(7 \tau_{W}^{2}\right) \\
A_{u_{s}}^{\gamma \gamma}=A\left(d_{u_{s}}\right)\left[\alpha B\left(3-d_{u_{s}}, 3\right) B\left(3-d_{u_{s}}, d_{u_{s}}\right)+\beta B\left(3-d_{u_{s}}, 4\right) B\left(4-d_{u_{s}}, d_{u_{s}}\right)\right] / \sin \left(\pi d_{u_{s}}\right)
\end{gathered}
$$


and

$$
\begin{aligned}
& A_{u_{f}}^{\gamma \gamma}=2 A\left(d_{u_{f}}-1 / 2\right)\left[\gamma B\left(5 / 2-d_{u_{f}}, 2\right) B\left(5 / 2-d_{u_{f}}, d_{u_{f}}-1 / 2\right)+\delta B(7 / 2-\right. \\
& \left.\left.d_{u_{f}}, 3\right) B\left(7 / 2-d_{u_{f}}, d_{u_{f}}-1 / 2\right)+\eta B\left(9 / 2-d_{u_{f}}, 4\right) B\left(9 / 2-d_{u_{f}}, d_{u_{f}}-1 / 2\right)\right] / \\
& \cos \left(\pi d_{u_{f}}\right) \\
A_{u_{s}}^{Z, \gamma}= & 2\left(2-d_{u_{s}}\right) A\left(d_{u_{s}}\right) \int_{0}^{1} d y \int_{0}^{1-y} d z 4 y z(1-y-z)^{1-d_{u_{s}} /\left[\operatorname { s i n } ( \pi d _ { u _ { s } } ) \left(1-4 \tau_{u_{s}} y z-\right.\right.} \\
& \lambda_{u_{s}} y(1-y)^{\left.\left.2-d_{u_{s}}\right)\right]}
\end{aligned}
$$

$$
\begin{aligned}
A_{u_{f}}^{Z, \gamma}= & 2 A\left(d_{u_{f}}-1 / 2\right) \int_{0}^{1} d y \int_{0}^{1-y} d z(1-y-z)^{\frac{3}{2}-d_{u_{f}}}\left[4\left(2-d_{u_{f}}\right)^{2}(1-4 y z)+\right. \\
& \left.4\left(3 / 2-d_{u_{f}}\right) y z\right] /\left[\cos \left(\pi d_{u_{f}}\right)\left(1-4 \tau_{u_{f}} y z-4 \lambda_{u_{f}} y(1-y)^{\frac{5}{2}-d_{u_{f}}}\right)\right]
\end{aligned}
$$

with

$$
\begin{gathered}
L(\tau)= \begin{cases}\arcsin ^{2} \sqrt{\tau} & \text { for } \tau \leq 1 \\
-\left[\ln \left(1+\sqrt{1-\tau^{-1}}\right) /\left(1-\sqrt{1-\tau^{-1}}\right)-i \pi\right]^{2} / 4 & \text { for } \tau>1\end{cases} \\
A_{W}^{Z \gamma}(x, y)=4\left[\Im_{1}(x, y)-\Im_{2}(x, y)\right] \\
A_{t o p}^{Z \gamma}(x, y)=4\left(3-s_{w}^{2} / c_{w}^{2}\right) \mathfrak{I}_{2}(x, y)+\left[(1+2 / x) s_{w}^{2} / c_{w}^{2}-(5+2 / x)\right] \mathfrak{J}_{1}(x, y) \\
\mathfrak{I}_{1}(x, y)=x y[x-y+x y[L(1 / x)-L(1 / y)]+x[g(1 / x)-g(1 / y)]] /\left[2(x-y)^{2}\right] \\
\mathfrak{I}_{2}(x, y)=-x y[L(1 / x)-L(1 / y)] /[2(x-y)], \quad g(x)=\sqrt{1 / x-1} \arcsin \sqrt{x}
\end{gathered}
$$

and

$$
\begin{gathered}
\gamma=\left(2-d_{u_{f}}\right)^{2}, \quad \delta=8\left[2\left(2-d_{u_{f}}\right)^{2}\left[\left(5 / 2-d_{u_{f}}\right) \tau_{u_{f}}-1\right]+\left(3 / 2-d_{u_{f}}\right)\right] \\
\beta=16\left(2-d_{u_{s}}\right)^{2} \tau_{u_{s}}, \quad \alpha=4\left(2-d_{u_{s}}\right), \\
\eta=32\left(5 / 2-d_{u_{f}}\right) \tau_{u_{f}}\left[-2\left(2-d_{u_{f}}\right)^{2}+\left(5 / 2-d_{u_{f}}\right)\right] \\
\tau_{i}=m_{h}^{2} / 4 m_{i}^{2}, \quad \lambda_{u_{i}}=M_{Z}^{2} / M_{u_{i}}^{2}, \quad g_{Z u_{i} u_{i}}=\left(T_{3}^{i}-Q_{u_{i}} s_{w}^{2}\right) /\left[c_{w} s_{w}\right]
\end{gathered}
$$

Here $s, t$ and $f$ stand for scalar, tensor and fermion unparticles. Concerning the ungauged unparticles, for the gluon-gluon fusion contribution, we have calculated the distributions as a function of the rapidity $y$, transverse momentum $P_{T}$ and the invariant mass $Q$ of the diphoton for a fixed choice of the LHC parameters. In fact, we have used the kinematical cut off given by ATLAS [3] which are the transverse momentum $P_{T}>25 \mathrm{GeV}$ and the rapidity $|y|<2.5$ for each photon. The electromagnetic and strong couplings constants are chosen such that $\alpha_{e m}=$ $1 / 128$ and $\alpha_{s}\left(M_{Z}\right)=0.118$. In all our numerical calculations, we have used the MSTW 2008 pdf's [4]. The factorization and renormalization scales $\mu_{F}$ and $\mu_{R}$ are taken such as: $\mu_{F}=\mu_{R}=$ $m_{t}=175 \mathrm{GeV}$. It is worth to mention that the contribution of the unparticles includes three free parameters namely the scale $\Lambda_{u}$, the coupling constants $\lambda_{i}$ 's and conformal scale dimensions $d_{u_{i}}$. Since the cross section of the signal is proportional to $\lambda_{i} / \Lambda_{u}^{d_{u_{i}}}$, it decreases rapidly as $d_{u_{i}}$ and/or $\Lambda_{u}$ increases. In what follow and as an illustration, we take $d_{u_{s}} \approx 1.001$, $d_{u_{t}} \approx 4.001$ and $\Lambda_{u}=1 \mathrm{TeV}$. In all our study, all the distributions are calculated for three illustrative values $\lambda_{i} / \Lambda_{u}^{d_{u_{i}}} \approx 0.01,0.005,0.001$ (in arbitrary units). We have determined the invariant mass distribution $d \sigma / d \Omega$ over the whole range of $Q \in[100,1000] \mathrm{GeV}$ and for the 
above values of the couplings constants. The unparticles effects were noticed in the large values of $Q$ regions where the SM background is reduced. This is expected since the unparticle distribution is proportional to $Q^{d_{u_{i}}}$. Moreover, the signal dominates over the SM contributions for a strong coupling $\lambda_{i} / \Lambda_{u}^{d_{u_{i}}} \approx 0.01$ and becomes more significant at the central region where $y=0$. For the scalar contribution, we notice that the signal is larger than the SM background for all values of $Q$, especially for smaller values where the contribution of the gluon-gluon fusion is significant. For the tensor case, the contribution is not as important as the scalar one. It starts deviating from the background when $Q>500 \mathrm{GeV}$ and becomes significant for larger values of $Q$. This suggests that the diphoton production is a tool to extract the scalar unparticles signals at smaller values of $Q$. Regarding the rapidity distributions for $-2.5<y<2.5$, we have made an integration over $Q$ in the range of $600<Q<1000 \mathrm{GeV}$ where the unparticle contribution is dominant. Similar behaviors have been obtained for the $P_{T}$ dependence distribution $d \sigma / d P_{T}$ over the kinematical region $50<P_{T}<100 \mathrm{GeV}$ where the unparticles contribution is visible for large values of $P_{T}$. For the photon-photon scattering and in the scalar case, we have chosen $\lambda_{s, \gamma}=\lambda_{s, \gamma}^{\prime}=0.2$. We have made an analysis with an ILC collider energy $\sqrt{s} \in[300,1000] \mathrm{GeV}$ and $\pi / 6 \leq \theta_{c m} \leq 5 \pi / 6\left(\theta_{c m}\right.$ is the center of mass scattering angle). We have noticed that the effects of the scalar unparticles are important for $\sqrt{s}>400 \mathrm{GeV}$ and $d_{u_{s}} \approx 1.01,1.99$ and become more significant for larger values of the beam energy. However for $d_{u_{s}} \approx 1.1,1.2,1.3,1.4$ the contribution is less important. Moreover, the results are strongly dependent on the choice of the initial states polarizations. In fact, the largest contribution was obtained for the helicity configuration $(+,-,+,-)$. Now, as a function of $\cos \theta_{\mathrm{cm}}$, the distribution was more important for $\theta_{c m}=\pi / 2$ with $95 \%$ confidence level or $\chi^{2} \geq 2.706$ corresponding approximatively to $d_{u_{s}} \approx 1.01$ and luminosity $L=100 \mathrm{fb}^{-1}$. For the tensor case and with $d_{u_{s}} \approx 4.01$, the unparticle contribution dominates that of the SM when $\sqrt{s}>$ $700 \mathrm{GeV}$ and it is strongly dependent on the choice of the scale $\Lambda_{u}$. It is important to point out that, the $\theta_{c m}$ dependence of the tensor unparticles cross section is completely different from that of the scalar ones. Regarding the Higgs diphoton decay with gauged unparticles loop, for the scalar case, a good improvement of the ratio $R_{\gamma \gamma}$ (a constructive interference) requires a negative value of the coupling constant $\lambda_{h u_{s}}$ since $A_{u_{s}}^{\gamma \gamma}$ and $A_{S M}^{\gamma \gamma}$ has the same negative sign. It is suppressed for larger values of $\mu_{s}$ because the factor $1 /\left(\mu_{s}^{2}\right)^{2-d_{u_{s}}}$ can not exceed 1 in the allowed interval $1<d_{u_{s}}<2$. The results show also that a significant contribution is obtained for large negative values of the coupling $\lambda_{h u_{s}}$ and becomes important when the parameters $\mu_{s}$ and $d_{u_{s}}$ increase. As an illustrative example, for $d_{u_{s}}=1.01$, the experimental value of $R_{\gamma \gamma}=$ 1.65 [5] is obtained if $0.5<\left|\lambda_{h u_{s}}\right|<2.5$ corresponding to a value of $\mu_{s} \in$ $\left[80 \mathrm{GeV}, 120 \mathrm{GeV}\right.$ ] and for $d_{u_{s}}=1.1$, if $0.5<\left|\lambda_{h u_{s}}\right|<2.5$ corresponding to $\mu_{s} \in$ [80 GeV, $90 \mathrm{GeV}]$. However, for $d_{u_{s}}>1.1$, one gets $\lambda_{h u_{s}}>2.5$. To overcome this problem and since the contribution of $R_{\gamma \gamma}$ increases with $\lambda_{h u_{s}} Q_{u_{s}}^{2} /\left(\mu_{s}^{2}\right)^{2-d_{u_{s}}}$, one has to take larger values of the unparticle charge $Q_{\mu_{s}}$. For $Q_{\mu_{s}}=2$ (instead of $Q_{\mu_{s}}=1$ ), the value of the coupling $\lambda_{h u_{s}}$ is reduced by a factor of 4 . In fact, for $d_{u_{s}}=1.01,1.1,1.2$, one gets $-0.2<\lambda_{h u_{s}}<-1,-0.3<$ $\lambda_{h u_{s}}<-1.3$ and $-0.8<\lambda_{h u_{s}}<-2.5$ respectively. The experimental value of $R_{\gamma \gamma}$ is also obtained for $d_{u_{s}}=1.3$ where $\mu_{s} \in[80 \mathrm{GeV}, 150 \mathrm{GeV}]$, however, for $d_{u_{s}}=1.4$, it can not be reached. Thus, if the measured value $R_{\gamma \gamma}=1.65$ is confirmed at the LHC, $d_{u_{s}}$ has to be constrained between 1.01 and 1.4 . For the spin $1 / 2$ case, the situation is completely different since for various values of $d_{u_{f}}$, different effects of $A_{u_{f}}^{\gamma \gamma}$ contribute leading to a positive or negative coupling $\lambda_{h u_{f}}$ (destructive or constructive interference). For example for $d_{u_{f}}=$ 1.5, 1.6, 1.7, $A_{u_{f}}^{\gamma \gamma}$ is positive and a constructive interference requires $\lambda_{h u_{f}}>0$, however for 
$d_{u_{f}}=1.8,1.9,2.0 \quad A_{u_{f}}^{\gamma \gamma}$ is negative and a constructive interference requires $\lambda_{h u_{f}}<0$. Furthermore, we have noticed that for $d_{u_{f}}=1.5$, getting the value $R_{\gamma \gamma}=1.65$ needs $2.5<$ $\lambda_{h u_{f}}<5.2$ where $\mu_{f} \in[80 \mathrm{GeV}, 150 \mathrm{GeV}]$. For $d_{u_{f}}=2$, one has $-3.6<\lambda_{h u_{f}}<-2$. Contrary to the diphoton production the ratio $R_{Z \gamma}$ depends on both signs of $Q_{u} g_{Z u u}$ and $\lambda_{h u_{i}}$. We remind that $Q_{u} g_{Z u u}<0$ in the case of $T=0, Y=2$ and $Q_{u} g_{Z u u}>0$ when $T=1 / 2$, $Y=1$. Thus, for a scalar constructive contribution where $\lambda_{h u_{s}}<0, A_{u_{S}}^{Z \gamma}<0$ and $A_{S M}^{Z \gamma}>0$, the quantity $\lambda_{h u_{s}} A_{u_{S}}^{Z \gamma} / A_{S M}^{Z \gamma}>0$. Concerning the fermionic contribution, the situation is a little bit delicate since the sign of the fermionic loop function $A_{u_{f}}^{Z \gamma}$ depends on the scale dimension $d_{u_{f}}$ wheras $A_{S M}^{Z \gamma}>0$. Thus, a good improvement in the decay mode $Z \gamma$ needs $Q_{u} g_{Z u u}<0$ corresponding to $S U(2)_{L}$ singlets unparticles states whereas suppression corresponds to $S U(2)_{L}$ doublets. Equivalently, one can say that the unparticles contribution to the decay modes $h \rightarrow \gamma \gamma$ and $h \rightarrow Z \gamma$ are positively (resp. negatively) correlated in the case where the unparticles states are $S U(2)_{L}$ singlets (resp. doublets).

\section{Discussion and Conclusion}

For the subprocess $g g \rightarrow \gamma \gamma$, we have shown the impact of the scalar and tensor contributions to various distributions at the LHC energies. We have estimated, the cross sections of the signal and background at a scale $\Lambda_{u}=1 \mathrm{TeV}$ and for $d_{u_{s}}=1.001$ as well as $d_{u_{t}}=4.001$. It turns out that within these choices, the effects of tensor unparticles are observable only in the region of a large diphoton invariant mass and transverse momentum for a coupling $\lambda / \Lambda_{u}^{d_{u}} \approx 1$. Furthermore, we have also noticed that the scalar unparticles contribution can lead to significant contributions of approximately one order of magnitude to the considered distributions all over the allowed range of the invariant mass, rapidity and transverse momentum. In particular, the effects of the scalar unparticles are more important at smaller values of $Q$ where the contribution of the box diagram predominates. Thus, experimentally, if we measure a reasonable pairs of photons with lower values of the invariant mass, the signature of the new physics will be that of the scalar unparticles. For the subprocess $\gamma \gamma \rightarrow \gamma \gamma$, at the ILC energies, we have also studied the effects of the scalar and tensor unparticles [6]. We can improve the ratio signal/background by choosing appropriate polarizations of the initial beam. For $\Lambda_{u}=1 \mathrm{TeV}$, the effects of the scalar unparticles lead to considerable deviations from SM with energies at $\sqrt{s}=500 \mathrm{GeV}$ and $1 \mathrm{TeV}$. For the case of tensor unparticles, the contribution decreases rapidly with the increase of the scale value $\Lambda_{u}$ and the effects are observable only for $\Lambda_{u} \leq 1 \mathrm{TeV}$. We have also investigated the decays modes $h \rightarrow \gamma \gamma$ and $h \rightarrow Z \gamma$ unduced by the loops of the charged scalar and spinor unparticles. Using the asymptotic forms of the vertices functions within the dimensional regularization procedure, general expressions of the amplitudes are reduced to simple integrals [7]. With the use of the Dyson subtraction method, the obtained amplitudes are shown to be gauge invariant. The most interesting implications were that the loop contributions of the $S U(2)_{L}$ singlets unparticles have an important impact on the Higgs phenomenology at the LHC and can explain the observed excess in the ratio $R_{\gamma \gamma}$ for the decay mode $h \rightarrow \gamma \gamma$ at ATLAS detector. In the case of scalar unparticles, a good improvement in the decay mode $h \rightarrow \gamma \gamma$ requires a negative coupling constant $\lambda_{h u_{s}}$, wheras for spin $1 / 2$ unparticles, depending on the scale dimension $d_{u_{f}}$, the coupling constant $\lambda_{h u_{f}}$ is either 
positive or negative due to the change in the sign of the fermionic loop function $A_{u_{f}}^{\gamma \gamma}$. We have also made a combined analysis of the decay modes $h \rightarrow \gamma \gamma$ and $h \rightarrow Z \gamma$ in the case where the unparticles carry quantum numbers such as weak isospin $\mathrm{T}$ and hypercharge Y of the SM. We have shown that the simultaneous measure of the decay widths in the above channels allows getting informations on the nature of these states. Fig.1, displays contours lines for various values of the ratios $R_{Z \gamma}$ and $R_{\gamma \gamma}=1.65$ in the plane $\mu_{s}-\lambda_{h u_{s}}$ for the case of the $S U(2)_{L}$ singlet states $(T=0, Y=2)$ corresponding to the $d_{u_{s}}=$ 1.01. Fig.2, shows the ratio $R_{Z \gamma}$ in the plane $T-Y$ for $R_{\gamma \gamma}=1.65, d_{u_{s}}=1.01$ and $\mu=$ $300 \mathrm{GeV}$.

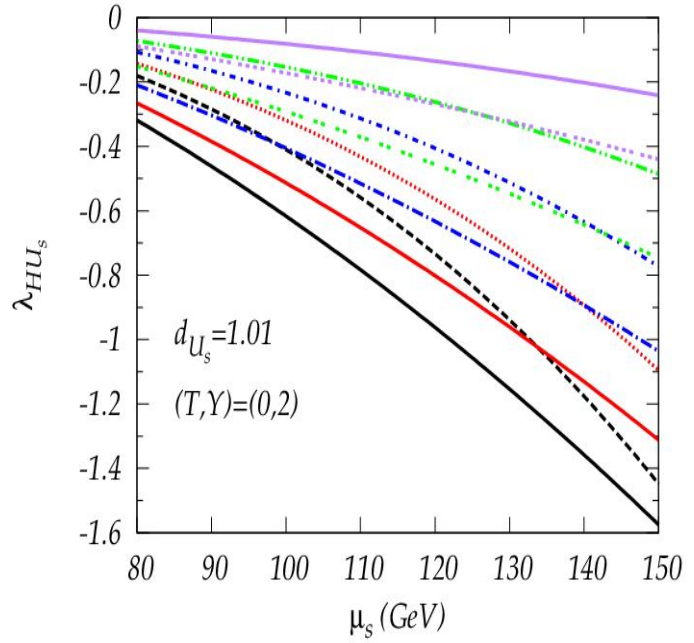

Fig1. Contour lines for various values of $R_{Z \gamma}$ for singlet states with $\boldsymbol{R}_{\gamma \gamma}=\mathbf{1 . 6 5}$ and $\boldsymbol{d}_{\boldsymbol{u}_{\boldsymbol{s}}}=\mathbf{1 . 0 1}$

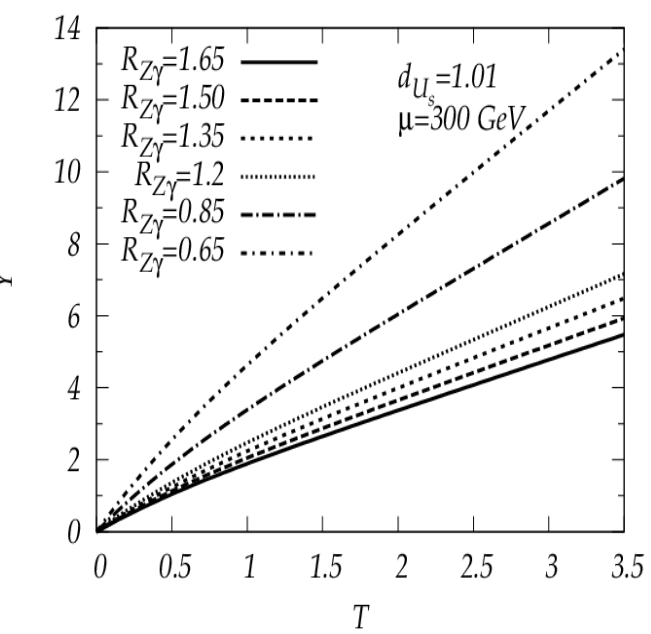

Fig2. Ratio $R_{Z \gamma}$ in the plane $T-Y$ for $R_{\gamma \gamma}=1.65$ $d_{u_{\mathrm{s}}}=1.01$ and $\mu=300 \mathrm{GeV}$

\section{References}

[1] H.Giorgi, Phys.Rev.Lett.98 (2007) 221601.

[2] S.L.Chen and X.G. He, Phys.Rev.D76 (2007) 091702.

[3] Atlas Collaboration, Atlas detector and physics performance. Technical design report. Vol.2 (1999), CERN-LHCC-99-15.

[4] A.D.Martin, W.J.Stirling,R.S.Thorne and .Watt, Eur.Phys.J.C63 (2009)189.

[5] G.Aad et al, [Atlas Collaboration], Phys.Lett.B,108 (2012)111803.

[6] I. Aliane, N. Mebarki, M. Haouchine in Helicity amplitudes of diphoton production at the LHC with scalar unparticles intermediate states AIP Conf.Proc. 1444 (2011) 171-174

[7] I. Aliane, N. Mebarki and Y. Delenda, Phys. Lett. B 728 (2014)549. 